\title{
Morphological alterations in dentine after mechanical treatment and ultrashort pulse laser irradiation
}

\author{
María Portillo Muñoz • María Cruz Lorenzo Luengo • \\ José Miguel Sánchez Llorente • Manuel Peix Sánchez • \\ Alberto Albaladejo • Ana García • Pablo Moreno Pedraz
}

Received: 6 December 2009 /Accepted: 4 October 2010 /Published online: 27 October 2010

(C) Springer-Verlag London Ltd 2010

\begin{abstract}
The aim of this study was to evaluate and compare the morphological changes that occur in dentine after femtosecond laser irradiation and after mechanical treatment. The duration of the laser pulse is an important parameter, because within the time frame of the pulse heat diffusion plays a very important role in the mechanism of interaction between the light and the tissue. Six totally impacted human third molars were sectioned into sheets approximately $1 \mathrm{~mm}$ thick with an Accutom-50 precision cutting machine. The samples were randomly divided into two groups according to their cavity preparation: mechanical cavity preparation and laser cavity preparation. The samples were then examined by light microscopy and scanning electron microscopy. There were clear differences in the results obtained with the two techniques. Cavities prepared with the laser with pulses of $<1$ ps showed no microcracks, and the treated surface displayed a rough and irregular aspect with no smear layer and exhibited open dentinal tubules. On the contrary, cavities made with a rotatory instrument had a smooth surface and microcracks, a broad area of carbonization and merging, occluded
\end{abstract}

\footnotetext{
M. Portillo Muñoz $\cdot$ M. C. Lorenzo Luengo •

J. M. Sánchez Llorente $(\triangle) \cdot$ M. Peix Sánchez $\cdot$ A. Albaladejo

Dental Clinic, Faculty of Medicine, University of Salamanca,

Paseo Universidad de Coimbra s/n,

37007 Salamanca, Spain

e-mail: jmsll@usal.es

P. Moreno Pedraz

Departamento de Ingeniería Mecánica, Faculty of Physics, University of Salamanca,

Salamanca, Spain

A. García $\cdot$ P. Moreno Pedraz

Grupo de Investigación en Láseres Intensos, Faculty of Physics, University of Salamanca,

Salamanca, Spain
}

dentinal tubules and a smear layer. This study showed that human dentine can be successfully ablated with the ultrashort pulse laser.

Keywords Femtosecond - Laser - Dentine - Thermal effects

\section{Introduction}

Rotary instruments have been used for cavity preparation and caries removal since the beginning of operative dentistry. Cavity extension is no longer used for prevention with the advances in new materials and techniques that allow minimally invasive dentistry. Lasers have become a valuable tool in several scientific fields, and in dentistry they are currently used to eliminate caries, in the treatment of dentine and enamel, to bleach the teeth, to sterilize root canals, and in soft-tissue surgery [1].

The characteristics of the titanium:sapphire laser (femtosecond laser) were first discussed by Moulton in 1986 [2]. Femtosecond lasers have also been used recently in dentistry on hard dental tissues [3, 4]. In recent years, the production of high-intensity laser sources has been an important line in the development of this technology, in particular high-intensity sources that produce extremely short pulses, below the picosecond range $\left(10^{-12} \mathrm{~s}\right)$. These laser pulses, amplified to energies of the order of millijoules and, when focused correctly, allow the ablation of surfaces with extreme precision and reproducibility, and as a result they cause much less collateral damage in the adjacent material than any other thermal, chemical or mechanical process [5].

Laser pulse duration is an important parameter because within the time frame of the pulse heat diffusion plays a very important role in the mechanism of interaction 
between the light and the tissue [6]. Shortening the pulses minimizes heating effects and allows the introduction of new mechanisms such as plasma-mediated ablation, which differs from the thermal ablation employed by conventional lasers in the almost total absence of thermal effects, which results in a significant increase in precision and quality of machining [7-12]. The mechanism basically consists of the ionization, by nonlinear processes, of atoms or molecules on the surface of the irradiated material, which form a dense plasma that expands upon completion of the pulse (duration in the order of $100 \mathrm{fs}$ ) with no time for heat diffusion to occur in the solid material (which requires pulses in the order of tens of picoseconds), resulting in the removal of material with very little heating [5].

Generally, when longer pulses are used more undesirable thermal effects appear since thermal energy accumulates and penetrates sufficiently deeply into the tissue to induce thermomechanical damage and, as a result, irreversible damage to the dental pulp tissue, which is particularly sensitive to such thermal effects. Moreover, in hard dental tissues microfractures or "cracks" are formed as a consequence of the increase in temperature. Shorter pulses reduce heat and at the same time diminish the effect of ablation; thus, shorter pulses can reduce heat and structural damage to tissues [13].

The aim of this study was to evaluate and compare the morphological changes that occur in dentine after femtosecond laser irradiation with different parameters and after mechanical treatment.

\section{Materials and methods}

Sample preparation

Six totally impacted human third molars, extracted over a period of 1 week, were collected at the Oral and Maxillofacial Surgery Unit of the Virgen de la Vega Hospital in Salamanca. The teeth were stored in an oven at $37^{\circ} \mathrm{C}$ for no longer than 1 month in physiological saline solution to prevent them from drying out. Subsequently, they were cut transversely into two parts: crown and root. Each crown was sectioned longitudinally in a vestibulolingual direction into sheets approximately $1 \mathrm{~mm}$ thick, using an Accutom-50 (Denmark, Copenhagen) precision cutting machine and Struers $330 \mathrm{~K}$ diamond grinding disks with abundant water as coolant. Approximately 9-11 sheets were obtained from each specimen. After discarding the sheets containing only enamel, 58 sheets were obtained. These samples were ground with sandpaper of 300, 400 and 600 grit in a polishing machine. Following this, the samples were kept in an oven at $37^{\circ} \mathrm{C}$ (for a maximum period of 1 month) in physiological saline solution to prevent them from drying out until the microscopy study. They were then randomly divided into two groups according to the cavity preparation method: mechanical cavity preparation $(n=29)$ and laser cavity preparation $(n=29)$. Each laser sample was treated using all investigated parameters (Table 1).

\section{Mechanical cavity preparation}

A Kavo handpiece was used to make $550-600 \mu \mathrm{m}$ perforations in the dentine samples at a speed of 450,000 rpm with a $0.5-\mathrm{mm}$ round tungsten bur (H1.314.005; Komet, Lemgo, Germany) with water as coolant.

\section{Laser cavity preparation}

The study was performed at the Laser Service of the University of Salamanca with a system based on a titanium:sapphire oscillator (Tsunami; Spectra-Physics, Mountain View, CA), which produces pulses of $100 \mathrm{fs}$, wavelengths in the near infrared region $(795 \mathrm{~nm})$, and energies of the order of $10 \mathrm{~nJ}$, with a repetition rate of $80 \mathrm{MHz}$. Even focusing with microscope objectives, these low-energy pulses are unable to ablate the surface of materials. In order to provide sufficient energy the pulses must be amplified with a "regenerative" system (Spitfire; Spectra-Physics) which is based on the chirped pulse amplification technique developed in the 1980s by Mourou and Strickland [14].

The samples were fixed in a precision X-Y-Z translator stage under computer control (Micos ES100; Nanotec, Munich, Germany). Horizontal XY movements allowed the area that we wished to structure to be swept, while the $\mathrm{Z}$ movement allowed pulses to be focused exactly on the

Table 1 Schematic representation of the parameters used

\begin{tabular}{llllll}
\hline \multirow{2}{*}{ Row } & Parameter & Column & & \\
\cline { 3 - 6 } & & 1 & 2 & 3 & 4 \\
\hline A & Pulse energy (mJ) & 0.05 & 0.05 & 0.05 & 0.05 \\
& Speed (mm/s) & 0.1 & 0.1 & 0.05 & 0.05 \\
& Steps (mm) & 0.02 & 0.01 & 0.01 & 0.02 \\
B & Pulse energy (mJ) & 0.03 & 0.03 & 0.03 & 0.03 \\
& Speed (mm/s) & 0.1 & 0.1 & 0.05 & 0.05 \\
& Steps (mm) & 0.02 & 0.01 & 0.01 & 0.02 \\
C & Pulse energy (mJ) & 0.01 & 0.01 & 0.01 & 0.01 \\
& Speed (mm/s) & 0.1 & 0.1 & 0.05 & 0.05 \\
& Steps (mm) & 0.02 & 0.01 & 0.01 & 0.02 \\
D & Pulse energy (mJ) & 0.007 & 0.007 & 0.007 & 0.007 \\
& Speed (mm/s) & 0.1 & 0.1 & 0.05 & 0.05 \\
& Steps (mm) & 0.02 & 0.01 & 0.01 & 0.02 \\
\hline
\end{tabular}


sample surface. The cavities were made in the dentine near the dentinoenamel junction. In order to obtain microstructures with a rectangular geometry, the samples were swept in parallel lines at different speeds $(v)$, pulse energies $(E)$ and steps $(s)$. The step is the movement of the laser beam between two shots. The different energies used in this study were as follows: $0.05 \mathrm{~mJ}$ (row A), $0.03 \mathrm{~mJ}$ (row B), $0.01 \mathrm{~mJ}$ (row C) and $0.007 \mathrm{~mJ}$ (row D) (Table 1). The cavities in columns 1 and 2 were obtained at a speed of $0.1 \mathrm{~mm} / \mathrm{s}$, and in columns 3 and 4 they were obtained at a speed of $0.05 \mathrm{~mm} / \mathrm{s}$ (Table 1). The steps were $0.02 \mathrm{~mm}$ for columns 1 and 4 and $0.01 \mathrm{~mm}$ for columns 2 and 3 (Table 1). No external water cooling system was used.

Analysis of samples

\section{Light microscopy}

All samples were examined under an Axio picture M1 (Carl Zeiss, Germany) light microscope. We used Epiplan $\times 20$ and $\times 50$ HD objectives (Carl Zeiss Vision), attached to a 1,300 $\times$ 1,030-pixel digital camera (Axiocam HR; Carl Zeiss Vision). The images obtained were processed with Axiovision software. This software measured the depth and diameter of the cavities made.

\section{Electron microscopy}

For viewing of samples under the scanning electron microscope (SEM), they were set up on aluminium disks and coated with a layer of gold using a SEM coating system from Bio-Rad. Images of the samples were obtained using a secondary electron detector in a DSM 940 Zeiss electron microscope at the Electron Microscopy Service of the University of Salamanca. Samples were viewed perpendicularly and multiple images were obtained at different magnifications of the most representative areas of each sample for subsequent assessment. These images were scanned directly onto a Hewlett-Packard XW 8000 workstation to allow comparison between samples. All images were saved in TIF format.

\section{Results}

The effects of the two cavity preparation techniques (mechanical preparation and laser preparation using the femtosecond laser at $795 \mathrm{~nm}$ ) on dentine were studied. First, we describe the results obtained for the laser cavity preparations and then we address those obtained with the mechanical system.

In brief, microscopic evaluation of the laser-prepared cavities showed that the depth of the craters appeared to increase with increasing energy and vice versa. There was no difference in the morphology of the cavities obtained with the same energies and steps when the sweep speed was varied. The larger step $(0.02 \mathrm{~mm})$ removed less dentine, since there were areas that were not affected by the radiation, presenting an intact dentine, and even dentine tubules were seen. However, with the $0.01 \mathrm{~mm}$ step, all the superficial dentine was removed without leaving areas of intact dentine.

Figure 1 shows a cavity obtained with the femtolaser with $E=0.007 \mathrm{~mJ}, v=0.05 \mathrm{~mm} / \mathrm{s}$ and $s=0.02 \mathrm{~mm}$. The cavity was a square of approximately $210 \mu \mathrm{m}$. Morphological analysis revealed a well-defined cavity surface, and intact dentine was present inside the cavity corresponding to the nonradiated dentine resulting from an increase in step. Additionally, the zone adjacent to the cavity had numerous open dentinal tubules with no smear layer. No signs of collateral damage such as carbonization, charring or cracks were observed.

When speed was increased and the step was decreased $(E=0.007 \mathrm{~mJ}, v=0.1 \mathrm{~mm} / \mathrm{s}$ and $s=0.01 \mathrm{~mm})$, the cavity (Fig. 2) was a square of approximately $200 \mu \mathrm{m}$ and showed no thermal effects. However, no irradiated dentine was observed inside the cavity. The bottom of the cavity showed a characteristic pattern of replacement of ejected material; this pattern was related to the direction of the laser beam.

The results obtained upon increasing the energy per pulse $(E=0.03 \mathrm{~mJ}, v=0.05 \mathrm{~mm} / \mathrm{s}$, and $s=0.02 \mathrm{~mm})$ are shown in Fig. 3. The cavity was a square of approximately $205 \mu \mathrm{m}$. The limits of the cavity were well defined and the cavity showed no cracks as a result of heat damage. Dentinal tubules remained open with no smear layer. Although the step was $0.02 \mathrm{~mm}$, no intact dentine was observed inside the microcavity; this was because of the increase in laser energy which caused a more pronounced

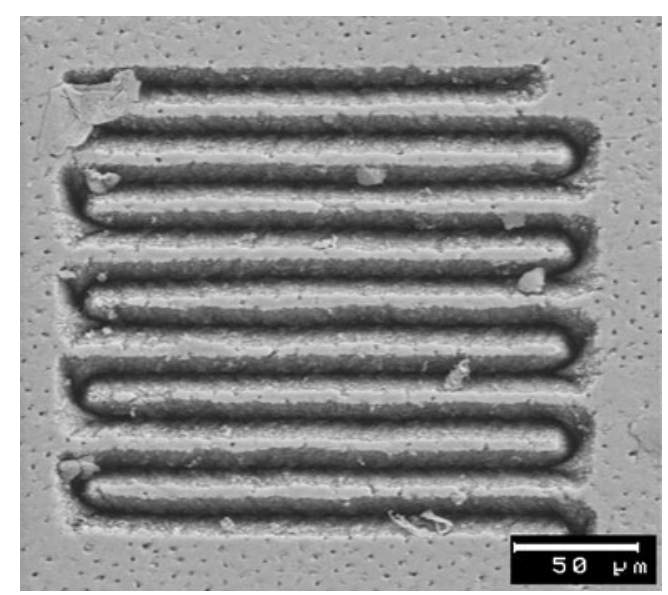

Fig. 1 SEM micrograph of a cavity in dentine prepared with the femtolaser at $E=0.007 \mathrm{~mJ}, v=0.05 \mathrm{~mm} / \mathrm{s}$ and $s=0.02 \mathrm{~mm}$ (original magnification $\times 374$ ) 


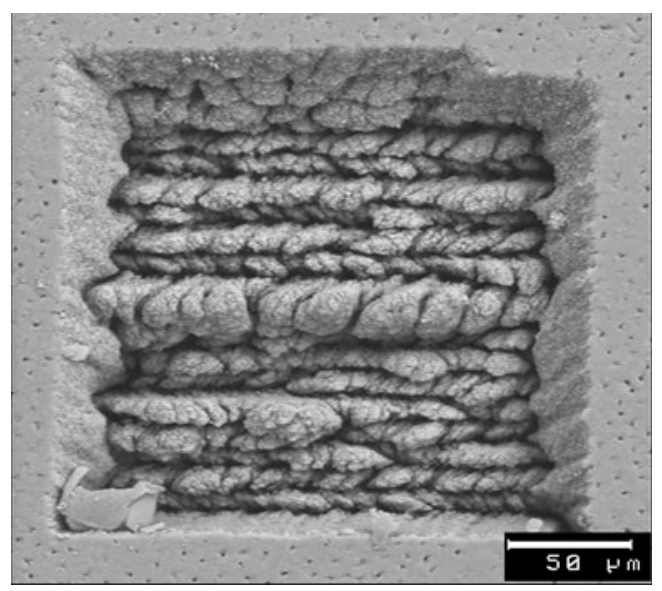

Fig. 2 SEM micrograph of a cavity in dentine prepared with the femtolaser at $E=0.007 \mathrm{~mJ}, v=0.1 \mathrm{~mm} / \mathrm{s}$ and $s=0.01 \mathrm{~mm}$ (original magnification $\times 374$ )

ablation of the dentine, and this in turn was responsible for the characteristic shape with higher areas alternating with deeper depressions.

In order to analyse the impact of step size on the morphology of the cavity, we compared the results obtained with $s=0.02 \mathrm{~mm}$ (Fig. 3) with those obtained with $s=0.01 \mathrm{~mm}$ (Fig. 4), keeping the other two parameters constant. Thus, as shown in Fig. 4 in a cavity made with the femtolaser at $E=0.03 \mathrm{~mJ}, v=0.1 \mathrm{~mm} / \mathrm{s}$ and $s=0.01$ of approximately $220 \mu \mathrm{m}$ square, the smaller step was associated with a greater degree of dentine ablation.

It is remarkable that the surface did not show cracks or signs of heat damage; however, the dentinal tubules were more occluded, probably as a result of the increased energy and the smaller step, but in this cavity the tubules were not

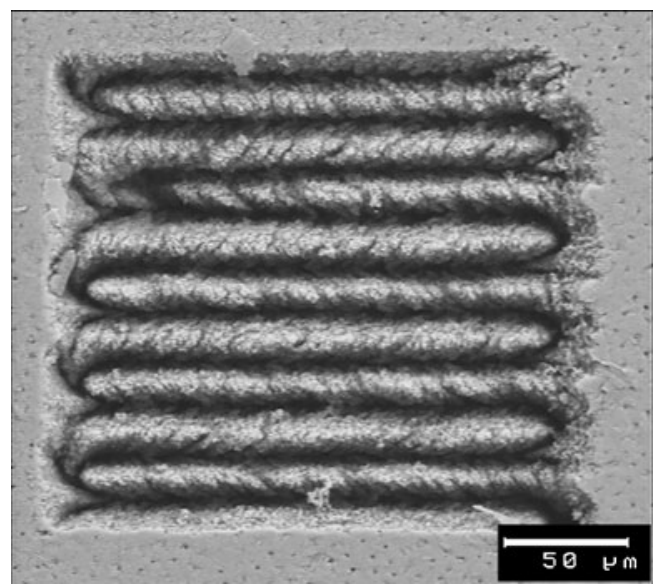

Fig. 3 SEM micrograph of a cavity in dentine prepared with the femtolaser at $E=0.03 \mathrm{~mJ}, v=0.05 \mathrm{~mm} / \mathrm{s}$ and $s=0.02 \mathrm{~mm}$ (original magnification $\times 374$ )

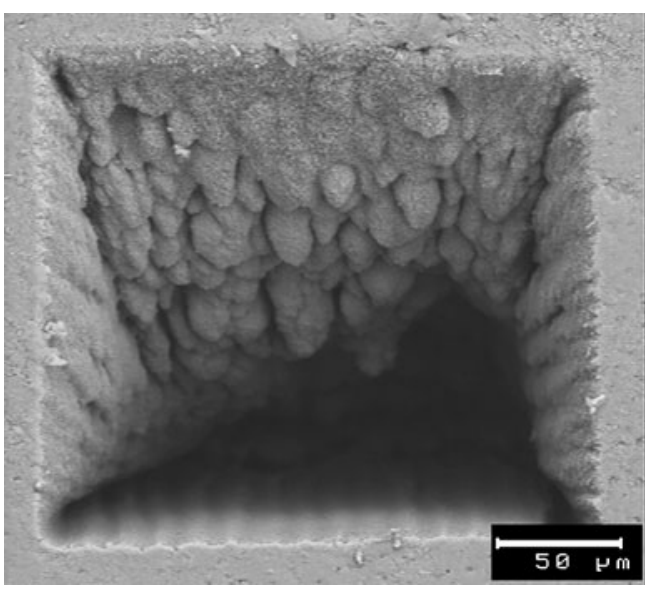

Fig. 4 SEM micrograph of a cavity in dentine prepared with the femtolaser at $E=0.03 \mathrm{~mJ}, v=0.1 \mathrm{~mm} / \mathrm{s}$ and $s=0.01 \mathrm{~mm}$ (original magnification $\times 374$ )

as sealed as in cavities obtained with the rotary instrument. In addition, an area of sedimentation of material was seen both at the bottom and the top of the cavity; this could correspond to the ablated dentine deposited at the bottom.

Figure 5 shows a cavity made with the rotatory instrument at a speed of 450,000 rpm under abundant water cooling. Circular cavities with a diameter between 560 and $600 \mu \mathrm{m}$ were made. The cavity shown in Fig. 5 has a smooth floor and walls and three cracks radiating outwards from the cavity, produced during cooling following the temperature increase caused by contact of the bur with the surface. The micrograph also reveals a broad area of carbonization and merging; in this case the dentinal tubules were occluded due to the evident presence of a smear layer.

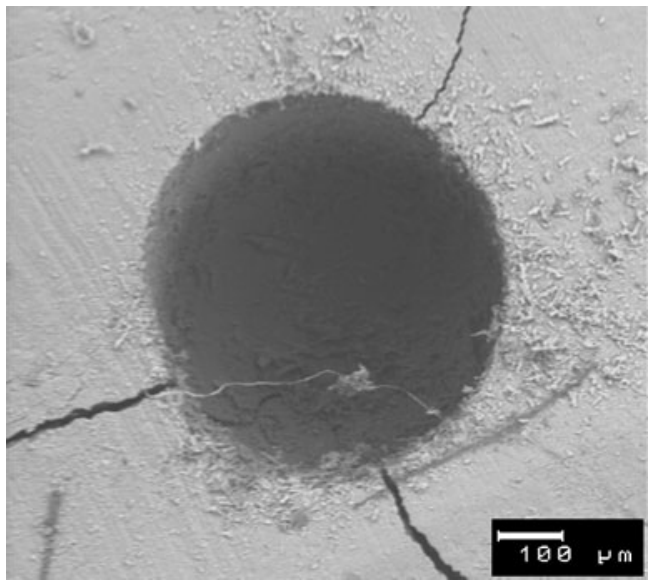

Fig. 5 SEM micrograph of a cavity in dentine prepared with the tungsten bur at a speed of $450.000 \mathrm{rpm}$ under abundant water cooling (original magnification $\times 100$ ) 


\section{Discussion}

In this study, a femtolaser was used for the ablation of human dentine. The laser parameters used were as follows: pulse energies $0.05,0.03,0.007$ and $0.01 \mathrm{~mJ}$; speeds 0.1 and $0.05 \mathrm{~mm} / \mathrm{s}$; and steps 0.02 and $0.01 \mathrm{~mm}$. Increasing or decreasing the speed did not influence either the morphology of the cavity or the effects of the laser on it; however, changing the step affected the morphological aspect of the cavity. Thus, by reducing the step from 0.02 to $0.01 \mathrm{~mm}$ a greater degree of dentine ablation was obtained and vice versa. This is logical because the number of times that the laser radiated the surface was doubled. In addition, when the energy was increased the volume of dentine removed also increased, but the morphology of the adjacent dentine was modified and a greater sealing of dentine tubules was observed. The dimensions of the cavities were not affected by these parameters, remaining at approximately at $200 \mu \mathrm{m}$ [13].

In all cases, dentine ablation was accompanied by minimal morphological changes in comparison with mechanical treatment. The limited number of studies that currently exist about hard dental tissue ablation with ultrashort lasers, similar to the laser used in our study, suggest that human dentine can be successfully ablated with these devices; furthermore, application of subpicosecond pulses almost completely prevents thermal damage and microcracking in neighbouring tissue, as observed here $[3,5,13,15,16]$. Moreover, studies on the application of these lasers in other tissues such as bone confirm that the temperature increases generated are low and result in only minor thermal and mechanical damage to the tissue [17-19].

The results of the present study suggest that to improve the quality of tooth treatment the application of ultrashort pulse lasers can offer an alternative to the classical mechanical technique.

It is well known that cutter remains are generated when mechanical instruments are used to prepare cavities, some of which inevitably become compacted to form a layer on the cutting surface (known as the smear layer). This material is made up of loosened particles of enamel, dentine and cement with a particle size between 1 and $50 \mu \mathrm{m}$, and the smaller particles can enter the lumen of the dentinal tubules and partially occlude them.

As in previous studies, our results obtained with the SEM suggest that the surface of cavities made with mechanical instruments is smooth, and the presence of a smear layer and occluded dentinal tubules are characteristic. Therefore, these cavities must subsequently be upgraded to provide anchorage for the restorative materials [20]. The superiority of femtosecond lasers for the precise machining of different materials has been demonstrated [5, 7, 21], attributing this to the fact that lasers produce minimum thermal and mechanical damage to the surface. This attribute makes the laser a good candidate for use in hard dental tissues since the absence of microcracks obtained with ultrashort pulses is of great importance in dentistry because such cracks may be a source of new caries. In addition, an intact interface acting as a barrier to the microleakage of bacteria and oral fluids is important in preventing dental pathology and pain $[4,16,22]$.

The friction produced by rotatory instruments generates high temperatures that cause irreversible damage to the tooth. The tooth surface shows signs of thermal and mechanical damage (cracks), and this technique leads to the formation of a smear layer. In contrast, the femtosecond (titanium:sapphire) laser causes minimal structural change to the irradiated dentine. The surfaces showed a complete absence of thermal and mechanical damage such as cracks, craters and charring. The treated surface had a rough and irregular aspect without a smear layer or cracks, and with open dentinal tubules, which would contribute to a greater strength of the restorative materials. These benefits have been also described by several other authors $[3,15,16]$.

The size and shape of burs do not allow complex shapes to be obtained or minimally invasive treatment [19]. A recent study has concluded that the use of lasers in cavity preparation involves a smaller loss of healthy dentine [23]. This is because the laser probably avoids the vibrations of mechanical instruments that reduce the precision of cutting and alter the morphology of the cavity. Lasers allow cavities ten times smaller than the smallest bur to be made $[17,18]$ and afford greater control over the removal of material.

Preparations made with mechanical instruments require simultaneous use of water as coolant to prevent damage to the pulp caused by high temperatures generated inside the cavity. This water coolant often reduces the visibility of the operative field. As seen here, by using ultrashort pulse lasers the need for irrigation is eliminated because the process of heating occurs over a shorter period than the normal time taken by the materials to transmit the heat (of the order of tens to hundreds of picoseconds). Additionally, the use of laser technology in operative dentistry reduces patient stress since there is no noise or need for anaesthesia for cavity completion.

A critical point for the application of femtolasers in practice is the time needed to treat a patient. Another drawback is that these lasers have not been currently marketed for use in dentistry and are still fairly expensive devices.

This study was an initial project aimed at determining the mechanical properties of laser-treated dentine when femtosecond pulses were used. Further studies will focus on the bonding of dental materials to laser-irradiated dentine.

Acknowledgments A.G. and P.M. acknowledge the support of Spanish Ministerio de Ciencia e Innovación through the Consolider Program SAUUL (CSD2007-00013) and research project FIS2009-09522, from 
Junta de Castilla y León through the Program for Groups of Excellence (GR27) and of the EC Seventh Framework Programme (LASERLABEUROPE, grant agreement no. 228334). We also acknowledge the support of the Centro de Laseres Pulsados, CLPU, Salamanca, Spain.

\section{References}

1. Youssef M, Quinelato A, Youssef F, Pelizon Pelino JE, Salvadori MC, Mori M (2008) Dentinal surface-cutting efficiency using a highspeed diamond bur, ultrasound and laser. Laser Phys 18:472-477

2. Moulton PF (1986) Spectroscopic and laser characteristics of Ti: Al2O3. J Opt Soc Am 3:125-133. doi:10.1364/JOSAB.3.000125

3. Kohns P, Zhou P, Stormann R (1997) Effective laser ablation of enamel and dentine without thermal side effects. J Laser Appl 9:171-174

4. Serbin J, Bauer T, Fallnich C, Kasenbacher A, Arnold WH (2002) Femtosecond lasers as novel tool in dental surgery. Appl Surf Sci 197-198:737-740

5. Niemz MH, Kasenbacher A, Strassl M, Backer A, Beyertt A, Nickel D, Giesen A (2004) Tooth ablation using a CPA-free thin disk femtosecond laser system. Appl Phys B 79:269-271

6. Rethfeld B, Sokolwski-Tinten K, von der Linde D, Anisimov SJ (2004) Timescales in the response of materials to femtosecond laser excitation. Appl Phys A 79:767-769

7. Chichkov BN, Momma C, Nolte S, von Alvensleben F, Tunnermann A (1996) Femtosecond, picosecond and nanosecond laser ablation solids. Appl Phys A 63:109-115

8. Momma C, Chichkov B, Nolte S, von Alvensleben F, Tünnermann A, Welling H (1996) Short-pulse laser ablation of solid targets. Opt Commun 129:134-142

9. Nolte S, Momma C, Jacobs H, Tunnermann A, Chichkov BN, Wellegehausen B, Welling H (1997) Ablation of metals by ultrashort laser pulses. J Optical Soc Am B 14:2716-2722

10. Pronko PP, Dutta SK, Squier SJ, Rudd JV, Du D, Mourou G (1995) Machining of sub-micron holes using a femtosecond laser at $800 \mathrm{~nm}$. Opt Commun 114:106-110

11. Stuart BC, Feit MD, Herman S, Rubenchik AM, Shore BW, Perry M (1996) Optical ablation by high-power short-pulse lasers. J Opt Soc Am B 13:459-468
12. Varel H, Ashkenasi D, Rosenfeld A, Wähmer M, Campbell EEB (1997) Micromachining of quartz with ultrashort laser pulses. Appl Phys A 65:367-373

13. Lizarelli RF, Costa MM, Carvalho-Filho E, Nunes FD, Bagnato VS (2007) Selective ablation of dental enamel and dentin using femtosecond laser pulses. Laser Phys Lett 5:63-69

14. Mourou G, Strickland D (1985) Compression of amplified chirped optical pulses. Opt Commun 55:447-449

15. Kruger J, Kautek W, Newesely H (1999) Femtosecond-pulse laser ablation of dental hydroxyapatite and single-crystalline fluoroapatite. Appl Phys A 69(Suppl):S403-S407

16. Pike P, Parigger C, Splinter R, Lockhart P (2007) Temperature distribution in dental tissue after interaction with femtosecond laser pulses. Appl Opt 46:8374-8378

17. Girard B, Cloutier M, Wilson DJ, Clokie CML, Miller RJD, Wilson BC (2007) Microtomographic analysis of healing of femtosecond laser bone calvarial wounds compared to mechanical instruments in mice with and without application of BMP-7. Lasers Surg Med 39:458-467

18. Girard B, Yu D, Armstrong MR, Wilson BC, Clokie CML, Dwayne RJ (2007) Effects of femtosecond laser irradiation on osseous tissues. Lasers Surg Med 39:273-285

19. Liu Y, Niemz M (2007) Ablation of femural bone with femtosecond laser pulses - a feasibility study. Lasers Med Sci 22:171-174. doi:10.1007/s10103-006-0424-8

20. Ekworapoj P, Sidhu SK, McCabe JF (2007) Effect of different power parameters of Er,Cr:YSGG laser on human dentine. Lasers Med Sci 22:175-182. doi:10.1007/s10103-006-0426-6

21. Rubenchik AM, Da Silva LB, Feit MD, Lane SM, London RA, Perry MD, Stuart BC, Neev J (1996) Dental tissue processing with ultrashort-pulsed laser. In: Wigdor HA (ed) Lasers in dentistry II. Proc SPIE 2672:222-230

22. Lizarelli RF, Kurachi C, Misoguti L, Bagnato VS (2000) A comparative study of nanosecond and picosecond laser ablation in enamel: morphological aspects. J Clin Laser Med Surg 18:151157

23. Eberhard J, Bode K, Hedderich J, Jepsen S (2008) Cavity size difference after caries removal by fluorescence-controlled Er:YAG laser and by conventional bur treatment. Clin Oral Investig 12:311-318 